\title{
Role of Radiation Therapy for Retropharyngeal Lymph Node Area in Hypopharyngeal Cancer
}

\author{
Mitsuru Okubo*, Tomohiro Itonaga, Tatsuhiko Saito, Sachika Shiraishi, Ryuji Mikami, Akira Sakurada, Shinji \\ Sugahara, Jinho Park and Koichi Tokuuye
}

Department of Radiology, Tokyo Medical University Hospital, Japan

Submission: May 30, 2018; Published: June 15, 2018

*Corresponding author: Mitsuru Okubo, Department of Radiology, Tokyo Medical University Hospital, 6-7-1 Nishishinjuku, Shinjuku-ku, Tokyo 1600023, Japan, Tel: +81-3-3342-6111; Email: okubo@tokyo-med.ac.jp

\begin{abstract}
Purpose: To retrospectively analyze the efficacy of irradiation of the retropharyngeal lymph node (RPLN) area in hypopharyngeal cancer without pretreatment RPLN metastasis.

Materials and Methods: We retrospectively identified patients with hypopharyngeal cancer irradiated curatively between May 2001 and August 2017. Patients in whom RPLN metastases were identified on pretreatment examination were excluded. The incidence of RPLN recurrence, and overall, progression-free survival probabilities were analyzed. Of a total of 90 consecutive patients who received curative radiotherapy at our institution, 11 patients (12\%) were positive for RPLN metastasis on pretreatment examination. Thus, 79 patients were included in the analysis. Of these 79 patients, 62 patients underwent radiotherapy to the whole neck including the RPLN area, and 17 patients to the whole neck excluding the RPLN area. The median follow-up duration was 35 months (range: 4-193 months).

Results: RPLN recurrence was identified in 1 patient (1.2\%). Overall survival probabilities at 5 years for patients irradiated to the whole neck including the RPLN area, and those excluding the RPLN area were $71 \%$ and $63 \%$, respectively. The difference in overall survival probabilities between patients irradiated to the whole neck including the RPLN area, and those excluding the RPLN area was not statistically significant $(p=0.314)$. Progression-free survival probabilities at 5 years for patients irradiated to the whole neck including the RPLN area, and those excluding the RPLN area were $45 \%$ and $52 \%$, respectively, and the difference was not statistically significant.
\end{abstract}

Conclusion: Irradiation for RPLN area in hypopharyngeal cancer without pretreatment RPLN metastasis may be unnecessary.

Keywords: Hypopharyngeal Cancer; Retropharyngeal Lymph Node; Irradiation; Rpln Recurrence; Overall And Progression-Free Survival Probabilities

Abbreviations: RPLN: Retropharyngeal Lymph Node; 3D-CRT: 3-Dimensional Conformal Radiotherapy; CT: Computed Tomography; MRI: Magnetic Resonance Imaging; SUV: Standardized Uptake Value; BSA: Body Surface Area; PET: Positron Emission Tomography

\section{Introduction}

Hypopharyngeal cancer is relatively rare, accounting for fewer than $5 \%$ of head and neck cancers [1]. The prognosis of hypopharyngeal cancer remains more unfavorable than that of most other types of cancer, owing to its late presentation, with $70 \%-90 \%$ of patients having stage III or IV disease at the time of presentation. This is attributable to the rapid lymphatic spread of these tumors and the anatomical location of the hypopharynx [2]. Patients with hypopharyngeal cancer have low 5-year survival rates of $15 \%-30 \%$ owing to the high risk of recurrence [3]. Surgery, radiotherapy, and/or chemoradiotherapy are selected as treatment options. Chemoradiotherapy protocols can achieve locoregional control of head and neck cancer [4,5]. Prophylactic irradiation of the whole neck is necessary in patients with hypopharyngeal cancer, owing to high rates of cervical lymph node metastases, with $40 \%$ of patients having contralateral occult nodal metastases early in the course of the disease $[3,6,7]$. Retropharyngeal lymph node (RPLN) metastasis is considered to be relatively common in patients with hypopharyngeal cancer. Investigators have reported that $13 \%-18 \%$ of patients had pathologically positive RPLNs in their surgical series [8-10]. The RPLN area is routinely defined as the radiotherapy target regardless of the clinical stage of the hypopharyngeal cancer $[11,12]$. However, it has been controversial whether performing radiotherapy to the RPLN area in patients with no evidence of RPLN metastasis has any beneficial effects. Some studies have been performed to analyze metastasis to the RPLN in patients with hypopharyngeal cancer who underwent pretreatment examinations. However, to our knowledge, the reports in the literature for RPLN recurrence after radiotherapy for hypopharyngeal cancer with no evidence of pretreatment RPLN 


\section{Global Journal of Otolaryngology}

metastasis are insufficient. Therefore, the purpose of this study was to retrospectively analyze the benefits of irradiation to the RPLN area in patients having hypopharyngeal cancer without pretreatment RPLN metastasis.

\section{Materials and Methods}

\section{Patients}

Ninety consecutive patients with hypopharyngeal cancer who were curatively treated using 3-dimensional conformal radiotherapy (3D-CRT) between May 2001 and August 2017 at Tokyo Medical University Hachioji Medical Center were retrospectively identified. All patients provided written informed consent, and this study was approved by the Ethical Review Board of the authors' institution. Patients in whom RPLN metastases were detected on pretreatment examination were excluded. Of a total of 90 consecutive patients who received curative radiotherapy at our institution, 11 patients $(12 \%)$ were positive for RPLN metastasis on pretreatment examination. Thus, 79 patients were included in this analysis. The patients' characteristics are summarized in Table 1. Seventy-six patients (96\%) were men and $3(4 \%)$ were women. The median age of the patients was 69 years (range, 39-90 years). Almost all patients (95\%) had an Eastern Cooperative Oncology Group performance status of 0 or 1 . The distribution of the primary site was as follows: piriform sinus in 53 patients, postcricoid area in 7 patients, and the posterior wall in 19 patients. More than $80 \%$ of the patients $(n=66,83 \%)$ had advanced-stage disease (stages III or IV).

Table 1: Patients' characteristics.

\begin{tabular}{|c|c|}
\hline No. of pts & 79 \\
\hline \multicolumn{2}{|l|}{ Sex (percentage) } \\
\hline Man & $76(96)$ \\
\hline Woman & $3(4)$ \\
\hline Age (years), median (range) & $69(39-90)$ \\
\hline \multicolumn{2}{|l|}{ Performance status (percentage) } \\
\hline 0 & $64(81)$ \\
\hline 1 & $10(13)$ \\
\hline 2 & $4(5)$ \\
\hline 3 & $1(1)$ \\
\hline \multicolumn{2}{|l|}{ Tumor site (percentage) } \\
\hline Piriform sinus & $53(67)$ \\
\hline Postcricoid area & $7(9)$ \\
\hline Posterior wall & $19(24)$ \\
\hline \multicolumn{2}{|l|}{ Clinical stage (percentage) } \\
\hline Stage I & $3(4)$ \\
\hline Stage II & $10(13)$ \\
\hline Stage III & $21(26)$ \\
\hline Stage IV & $45(57)$ \\
\hline No. of pts performed chemotherapy (percentage) & $57(72)$ \\
\hline Induction & $18(23)$ \\
\hline concomitant & $54(68)$ \\
\hline \multicolumn{2}{|l|}{ No. of pts irradiated for whole neck (percentage) } \\
\hline including RPLN area & $62(78)$ \\
\hline excluding RPLN area & $17(22)$ \\
\hline
\end{tabular}

RPLN, retropharyngeal lymph node

Determination of the level of the cervical lymph nodes by imaging was in accordance with the Radiation Oncology Group consensus guidelines based on the Danish Head and Neck Cancer Group, European Organization for Research and Treatment of Cancer, Grouped' Oncologie Radiotherapie Tete et Cou, and National Cancer Institute of Canada [13]. Cervical lymph nodes with a shortest axial diameter of $\geq 10 \mathrm{~mm}$, and retropharyngeal lymph nodes with a shortest axial diameter of $\geq 5 \mathrm{~mm}$ on computed tomography (CT) or magnetic resonance imaging (MRI) were defined as malignant. Lymph nodes of borderline size with abnormal enhancement were also determined as malignant $[14,15]$. Lymph nodes with a maximum fludeoxyglucose activity of $\geq 2.5$ standardized uptake value (SUV) were also regarded as malignant [16-18]. The disease stage was determined in accordance with the 2002 TNM classification $\left(6^{\text {th }}\right.$ edition, International Union Against Cancer). 


\section{Global Journal of Otolaryngology}

\section{Treatments}

The 3D-CRT treatment was planned and performed with the patient using a shell in the supine position. For treatment planning, all patients underwent cervical CT at a $2.5 \mathrm{~mm}$ slice thickness. Typically, the whole neck was irradiated at a dose of 40Gy, and an additional dose of 20-30Gy was administered to the primary site and the lymph node metastases. The radiotherapy field of the whole neck included areas of levels II, III, and IV, the supraclavicular lymph nodes, and IB or V with or without the RPLN area. Typical fields of whole neck irradiation both including and excluding the RPLN area are shown in Figure 1. Patients were treated with photons of $4 \mathrm{MV}$ and 2.0Gy, once a day, for 5 days a week. Induction and concomitant chemotherapy were performed on patients with stage IV and those with stage II or higher disease, respectively. Patients were not treated with chemotherapy if they had renal dysfunction or an Eastern Cooperative Oncology Group performance status of 2 or more.
From 2001 to 2013 , docetaxel hydrate $(80 \mathrm{mg} / \mathrm{m} 2$ body surface area [BSA]), cisplatin $(80 \mathrm{mg} / \mathrm{m} 2 \mathrm{BSA})$, and 5 -fluorouracil $(4,000 \mathrm{mg} / \mathrm{m} 2 \mathrm{BSA})$ were used as an induction chemotherapy 4 weeks before the start of radiotherapy, and they were used as a concomitant chemotherapy every 4 weeks during the radiotherapy. From 2014, induction chemotherapy consisted of docetaxel hydrate $(60 \mathrm{mg} / \mathrm{m} 2 \mathrm{BSA})$, cisplatin $(60 \mathrm{mg} / \mathrm{m} 2 \mathrm{BSA})$, and 5 -fluorouracil $(3,000 \mathrm{mg} / \mathrm{m} 2$ BSA $)$ administered 4 weeks before the start of radiotherapy. As a concomitant chemotherapy, either cisplatin or cetuximab was administered. Cisplatin $(80 \mathrm{mg} /$ m2 BSA) was administered every 3 weeks during radiotherapy. Cetuximab was administered at $400 \mathrm{mg} / \mathrm{m} 2$ BSA 1 week before the start of radiotherapy, and at a regular weekly dose of $250 \mathrm{mg} / \mathrm{m} 2$ BSA during radiotherapy. In summary, 57 patients $(72 \%)$ received induction or concomitant chemotherapy, and 62 patients $(78 \%)$ were irradiated to the whole neck, including the RPLN area. The median follow-up duration of the patients was 35 months (range: 4-193 months).

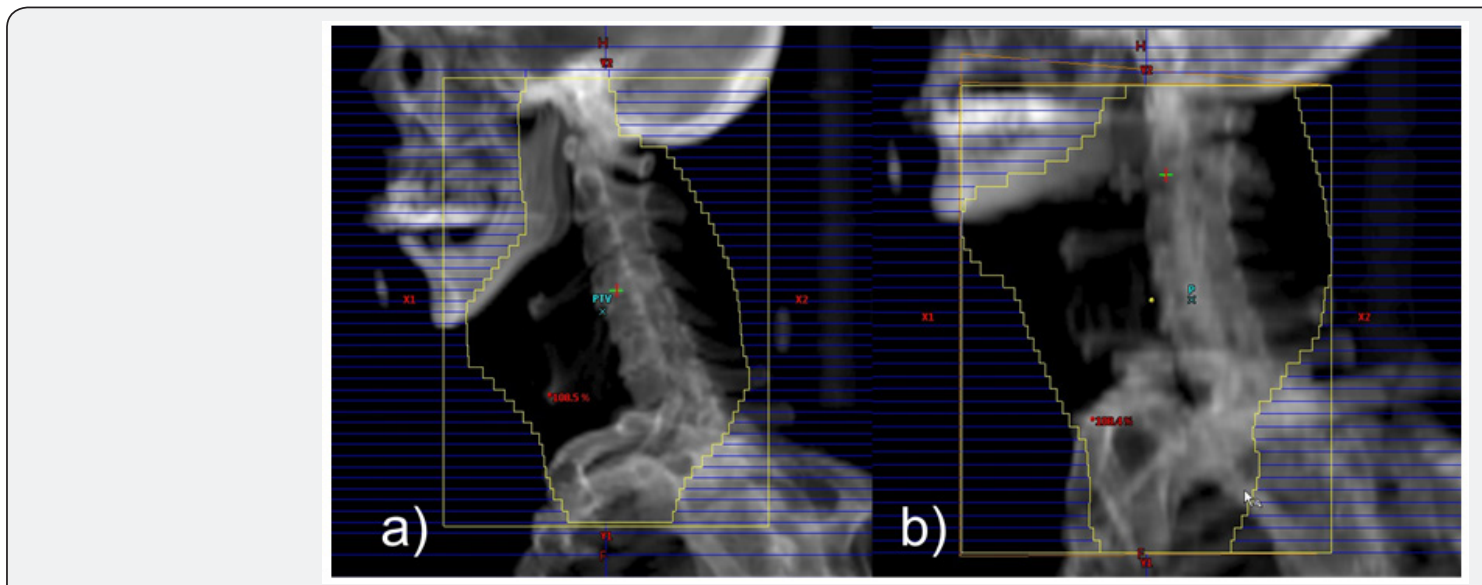

(a) A typical field of whole neck irradiation, including the RPLN area. (b) A typical field of whole neck irradiation, excluding the RPLN area.

Figure 1: Beam's eye views of radiation treatment planning

\section{Follow-up procedures}

Regular follow-up visits were performed once a month after completing radiotherapy for the first year, at 3 to 4 month intervals for the second and third years, and every 4 to 6 months thereafter, in the case of the absence of clinical symptoms. At each follow-up visit, the evaluation consisted of laryngoscopy, and medical history and physical examination. CT was performed every 6 months, and positron emission tomography-computed tomography (PET/CT) was performed once a year.

\section{Statistical Analysis}

The Fisher exact test was used to analyze differences in the incidences of RPLN recurrence between patients irradiated to the whole neck, including the RPLN area, and those excluding the RPLN area. The mean dose to the parotid glands was measured and compared between patients irradiated to the whole neck, including the RPLN area, and those excluding the RPLN area, using the Mann-Whitney U test. Survival curves were plotted using the Kaplan-Meier method, with statistical significance assessed by the log-rank test. Overall survival was defined as the time from the first day of radiotherapy to death due to any cause. Progression-free survival was defined as the time from the first day of radiotherapy to disease progression or death due to any cause. Overall and progression-free survival probabilities were compared between patients irradiated to the whole neck, including the RPLN area, and those excluding the RPLN area. Univariate logistic regression analyses were performed to analyze data using IBM SPSS Statistics 20.0 software (SPSS, Armonk, New York). A p-value of less than 0.05 was considered to indicate a statistically significant difference between 2 groups.

\section{Results}

\section{RPLN recurrence and survival outcomes}

The incidence of RPLN recurrence and mean dose of radiation to the parotid glands of patients irradiated to the whole neck, including the RPLN area, and those excluding the RPLN area 


\section{Global Journal of Otolaryngology}

are shown in Table 2. RPLN recurrence was identified in 1(1.2 $\%)$ of the 79 patients. This patient had received radiotherapy alone to the whole neck excluding the RPLN area, because of her advanced age (87 years) and weak condition (Performance status: 2). RPLN recurrence was identified on PET/CT at 7 months after radiotherapy. Information regarding the mean dose of irradiation to the parotid glands was available for 72 patients. The median mean dose of radiation to the parotid glands of patients irradiated to the whole neck, including the RPLN area, and those excluding the RPLN area were 37Gy (range: 24-43Gy) and 14Gy (1-27Gy), respectively, which showed a statistically significant difference $(\mathrm{p}<0.001)$ (Table 3$)$.

Table 2: The incident of RPLN recurrence and mean dose of parotid glands.

\begin{tabular}{|c|c|c|c|}
\hline & Patients irradiated for whole neck & p \\
\hline & Including RPLN & Excluding RPLN & \\
\hline RPLN recurrence & 0 & 1 & 0.2 \\
$\mathrm{n}=79$ & $\mathrm{n}=62$ & $\mathrm{n}=17$ & \\
\hline Mean dose of parotid glands & $37 \mathrm{~Gy}$ & $14 \mathrm{~Gy}$ & \\
Median (range) & $(24-43 \mathrm{~Gy})$ & $\mathrm{n}=17$ & \\
$\mathrm{n}=72$ & $\mathrm{n}=55$ & 0.001 & \\
\hline
\end{tabular}

RPLN, retropharyngeal lymph node

Table 3: Clinical data for all cases.

\begin{tabular}{|c|c|c|c|c|c|c|c|c|c|c|}
\hline No & Age & PS & $\mathbf{T}$ & $\mathbf{N}$ & M & Stage & Tumor site & Total dose & $\begin{array}{l}\text { Irradiation of whole } \\
\text { neck }\end{array}$ & $\begin{array}{l}\text { Mean dose of } \\
\text { parotid grand }\end{array}$ \\
\hline 1 & 66 & 1 & 3 & 0 & 0 & 3 & Piriform sinus & 70Gy/35fr & including RPLN area & - \\
\hline 2 & 65 & 0 & $4 a$ & $2 \mathrm{~b}$ & 0 & $4 a$ & Piriform sinus & 70Gy/35fr & including RPLN area & - \\
\hline 3 & 77 & 0 & 3 & 0 & 0 & 3 & Posterior wall & 70Gy/35fr & including RPLN area & - \\
\hline 4 & 77 & 0 & 3 & 0 & 0 & 3 & Piriform sinus & 68Gy/34fr & including RPLN area & - \\
\hline 5 & 79 & 1 & 3 & 1 & 0 & 3 & Piriform sinus & 70Gy/35fr & including RPLN area & - \\
\hline 6 & 47 & 0 & 2 & $2 c$ & 0 & $4 a$ & Piriform sinus & $60 \mathrm{~Gy} / 30 \mathrm{fr}$ & including RPLN area & - \\
\hline 7 & 70 & 0 & 2 & 1 & 0 & 3 & Piriform sinus & $60 \mathrm{~Gy} / 30 \mathrm{fr}$ & including RPLN area & - \\
\hline 8 & 83 & 1 & 3 & $2 \mathrm{~b}$ & 0 & $4 a$ & Piriform sinus & $54 \mathrm{~Gy} / 18 \mathrm{fr}$ & excluding RPLN area & 6Gy \\
\hline 9 & 70 & 0 & 3 & 1 & 0 & 3 & Piriform sinus & 66.6Gy/37fr & including RPLN area & 37Gy \\
\hline 10 & 70 & 0 & 2 & $2 \mathrm{~b}$ & 0 & $4 a$ & Piriform sinus & 60Gy/30fr & including RPLN area & 37 Gy \\
\hline 11 & 60 & 0 & 2 & 1 & 0 & 3 & Postcricoid area & $60 \mathrm{~Gy} / 30 \mathrm{fr}$ & including RPLN area & 37Gy \\
\hline 12 & 60 & 0 & 2 & $2 \mathrm{~b}$ & 0 & $4 a$ & Piriform sinus & $60 \mathrm{~Gy} / 30 \mathrm{fr}$ & including RPLN area & 39Gy \\
\hline 13 & 68 & 2 & 2 & 1 & 0 & 3 & Posterior wall & 66Gy/33fr & including RPLN area & 40Gy \\
\hline 14 & 74 & 0 & 2 & 1 & 0 & 3 & Piriform sinus & $64 \mathrm{~Gy} / 32 \mathrm{fr}$ & including RPLN area & 38Gy \\
\hline 15 & 78 & 0 & $4 a$ & 0 & 0 & $4 a$ & Posterior wall & 70Gy/35fr & including RPLN area & 39Gy \\
\hline 16 & 71 & 0 & $4 a$ & 1 & 0 & $4 a$ & Posterior wall & $60 \mathrm{~Gy} / 30 \mathrm{fr}$ & including RPLN area & 38Gy \\
\hline 17 & 58 & 0 & $4 a$ & $2 c$ & 0 & $4 a$ & Posterior wall & 70Gy/35fr & including RPLN area & 38Gy \\
\hline 18 & 69 & 0 & 2 & 1 & 0 & 3 & Piriform sinus & $60 \mathrm{~Gy} / 30 \mathrm{fr}$ & including RPLN area & 38Gy \\
\hline 19 & 66 & 0 & $4 \mathrm{a}$ & $2 \mathrm{~b}$ & 0 & $4 a$ & Piriform sinus & $60 \mathrm{~Gy} / 30 \mathrm{fr}$ & including RPLN area & 39Gy \\
\hline 20 & 76 & 0 & 2 & $2 \mathrm{~b}$ & 0 & $4 a$ & Piriform sinus & $60 \mathrm{~Gy} / 30 \mathrm{fr}$ & including RPLN area & 39Gy \\
\hline 21 & 61 & 0 & 3 & $2 \mathrm{~b}$ & 0 & $4 a$ & Piriform sinus & $60 \mathrm{~Gy} / 30 \mathrm{fr}$ & including RPLN area & 38Gy \\
\hline 22 & 80 & 0 & $4 a$ & $2 \mathrm{~b}$ & 0 & $4 a$ & Piriform sinus & 66Gy/33fr & including RPLN area & 39Gy \\
\hline 23 & 58 & 0 & $4 \mathrm{a}$ & 1 & 0 & $4 a$ & Posterior wall & $60 \mathrm{~Gy} / 30 \mathrm{fr}$ & including RPLN area & 37Gy \\
\hline 24 & 65 & 0 & $4 a$ & $2 \mathrm{~b}$ & 0 & $4 a$ & Postcricoid area & $60 \mathrm{~Gy} / 30 \mathrm{fr}$ & including RPLN area & 39Gy \\
\hline 25 & 57 & 0 & $4 a$ & $2 \mathrm{a}$ & 0 & $4 \mathrm{a}$ & Piriform sinus & 60Gy/30fr & including RPLN area & 38Gy \\
\hline 26 & 84 & 1 & 1 & $2 \mathrm{~b}$ & 0 & $4 a$ & Piriform sinus & 66Gy/33fr & including RPLN area & 39Gy \\
\hline 27 & 70 & 0 & $4 a$ & $2 \mathrm{c}$ & 0 & $4 a$ & Piriform sinus & 58Gy/29fr & including RPLN area & $37 \mathrm{~Gy}$ \\
\hline 28 & 53 & 0 & 2 & $2 \mathrm{~b}$ & 0 & $4 a$ & Piriform sinus & 60Gy/30fr & including RPLN area & 39Gy \\
\hline 29 & 70 & 0 & 2 & 0 & 0 & 2 & Piriform sinus & 60Gy/30fr & including RPLN area & 36Gy \\
\hline
\end{tabular}




\section{Global Journal of Otolaryngology}

\begin{tabular}{|c|c|c|c|c|c|c|c|c|c|c|}
\hline 30 & 80 & 0 & 2 & 0 & 0 & 2 & Piriform sinus & $60 \mathrm{~Gy} / 30 \mathrm{fr}$ & including RPLN area & $37 \mathrm{~Gy}$ \\
\hline 31 & 73 & 0 & 2 & 0 & 0 & 2 & Piriform sinus & $60 \mathrm{~Gy} / 30 \mathrm{fr}$ & including RPLN area & $37 \mathrm{~Gy}$ \\
\hline 32 & 75 & 1 & 3 & $2 \mathrm{~b}$ & 0 & $4 a$ & Piriform sinus & 60Gy/30fr & including RPLN area & 38Gy \\
\hline 33 & 72 & 3 & 2 & $2 \mathrm{a}$ & 0 & $4 \mathrm{a}$ & Piriform sinus & 66Gy/33fr & including RPLN area & 43Gy \\
\hline 34 & 53 & 0 & 2 & 0 & 0 & 2 & Piriform sinus & $60 \mathrm{~Gy} / 30 \mathrm{fr}$ & excluding RPLN area & $27 \mathrm{~Gy}$ \\
\hline 35 & 55 & 0 & 2 & 3 & 0 & $4 \mathrm{~b}$ & Posterior wall & $60 \mathrm{~Gy} / 30 \mathrm{fr}$ & including RPLN area & 35Gy \\
\hline 36 & 90 & 1 & 3 & 0 & 0 & 3 & Piriform sinus & 60Gy/30fr & excluding RPLN area & $1 \mathrm{~Gy}$ \\
\hline 37 & 63 & 0 & 4 & 3 & 0 & $4 \mathrm{~b}$ & Piriform sinus & 66Gy/33fr & including RPLN area & 31Gy \\
\hline 38 & 71 & 2 & 2 & 0 & 2 & 2 & Posterior wall & $60 \mathrm{~Gy} / 30 \mathrm{fr}$ & including RPLN area & 36Gy \\
\hline 39 & 87 & 0 & 3 & 1 & 0 & 3 & Posterior wall & $52 \mathrm{~Gy} / 26 \mathrm{fr}$ & excluding RPLN area & $1 \mathrm{~Gy}$ \\
\hline 40 & 70 & 0 & 3 & 1 & 0 & 3 & Posterior wall & 50Gy/25fr & including RPLN area & 37Gy \\
\hline 41 & 71 & 0 & 4 & $2 b$ & 0 & $4 a$ & Posterior wall & $60 \mathrm{~Gy} / 30 \mathrm{fr}$ & including RPLN area & 34Gy \\
\hline 42 & 65 & 0 & 2 & 1 & 0 & 3 & Posterior wall & 60Gy/30fr & including RPLN area & 36Gy \\
\hline 43 & 63 & 0 & 3 & 0 & 0 & 3 & Posterior wall & 60Gy/30fr & including RPLN area & 37Gy \\
\hline 44 & 73 & 0 & 2 & 1 & 0 & 3 & Piriform sinus & $60 \mathrm{~Gy} / 30 \mathrm{fr}$ & including RPLN area & 33Gy \\
\hline 45 & 57 & 0 & 2 & $2 b$ & 0 & $4 \mathrm{a}$ & Postcricoid area & $60 \mathrm{~Gy} / 30 \mathrm{fr}$ & including RPLN area & 38Gy \\
\hline 46 & 84 & 0 & $4 a$ & $2 \mathrm{c}$ & 0 & $4 \mathrm{a}$ & Piriform sinus & 66Gy/33fr & including RPLN area & $37 \mathrm{~Gy}$ \\
\hline 47 & 82 & 1 & 3 & 0 & 0 & 3 & Postcricoid area & 66Gy/33fr & including RPLN area & 30Gy \\
\hline 48 & 69 & 1 & $4 a$ & $2 \mathrm{a}$ & 0 & $4 \mathrm{a}$ & Piriform sinus & $54 \mathrm{~Gy} / 27 \mathrm{fr}$ & including RPLN area & $37 \mathrm{~Gy}$ \\
\hline 49 & 67 & 0 & 2 & 1 & 0 & 3 & Piriform sinus & $60 \mathrm{~Gy} / 30 \mathrm{fr}$ & including RPLN area & $37 \mathrm{~Gy}$ \\
\hline 50 & 76 & 0 & 1 & 0 & 0 & 1 & Piriform sinus & 66Gy/33fr & excluding RPLN area & 14Gy \\
\hline 51 & 52 & 0 & 2 & 0 & 0 & 2 & Postcricoid area & 60Gy/30fr & including RPLN area & 33Gy \\
\hline 52 & 67 & 0 & 1 & $2 \mathrm{a}$ & 0 & $4 \mathrm{a}$ & Piriform sinus & 66Gy/33fr & excluding RPLN area & $25 \mathrm{~Gy}$ \\
\hline 53 & 74 & 0 & 2 & 1 & 0 & 3 & Postcricoid area & 66Gy/33fr & including RPLN area & 36Gy \\
\hline 54 & 69 & 0 & $4 b$ & 1 & 0 & $4 \mathrm{~b}$ & Posterior wall & 60Gy/30fr & including RPLN area & 32Gy \\
\hline 55 & 87 & 2 & 3 & $2 \mathrm{~b}$ & 0 & $4 \mathrm{a}$ & Piriform sinus & $60 \mathrm{~Gy} / 30 \mathrm{fr}$ & excluding RPLN area & 15Gy \\
\hline 56 & 61 & 0 & 2 & $2 c$ & 0 & $4 a$ & Piriform sinus & $60 \mathrm{~Gy} / 30 \mathrm{fr}$ & including RPLN area & $37 \mathrm{~Gy}$ \\
\hline 57 & 64 & 0 & $4 a$ & 3 & 0 & $4 \mathrm{~b}$ & Piriform sinus & 70Gy/35fr & excluding RPLN area & 24Gy \\
\hline 58 & 68 & 0 & 1 & 0 & 0 & 1 & Piriform sinus & $70 \mathrm{~Gy} / 35 \mathrm{fr}$ & excluding RPLN area & 16Gy \\
\hline 59 & 59 & 0 & $4 a$ & $2 \mathrm{~b}$ & 0 & $4 a$ & Piriform sinus & $60 \mathrm{~Gy} / 30 \mathrm{fr}$ & including RPLN area & $37 \mathrm{~Gy}$ \\
\hline 60 & 62 & 0 & $4 a$ & 1 & 0 & $4 \mathrm{a}$ & Piriform sinus & $60 \mathrm{~Gy} / 30 \mathrm{fr}$ & including RPLN area & 24Gy \\
\hline 61 & 49 & 0 & 2 & 3 & 0 & $4 \mathrm{~b}$ & Piriform sinus & 60Gy/30fr & including RPLN area & 36Gy \\
\hline 62 & 62 & 0 & 2 & $2 \mathrm{~b}$ & 0 & $4 a$ & Piriform sinus & 66Gy/33fr & excluding RPLN area & 19Gy \\
\hline 63 & 55 & 1 & $4 a$ & $2 b$ & 0 & $4 a$ & Piriform sinus & 66Gy/33fr & including RPLN area & 39Gy \\
\hline 64 & 80 & 0 & 2 & $2 \mathrm{c}$ & 0 & $4 \mathrm{a}$ & Piriform sinus & 70Gy/35fr & excluding RPLN area & 24Gy \\
\hline 65 & 62 & 0 & 3 & 0 & 0 & 3 & Piriform sinus & 66Gy/33fr & excluding RPLN area & 9Gy \\
\hline 66 & 67 & 0 & 3 & $2 \mathrm{~b}$ & 0 & $4 a$ & Piriform sinus & 66Gy/33fr & excluding RPLN area & 22Gy \\
\hline 67 & 39 & 0 & 3 & $2 c$ & 0 & $4 a$ & Piriform sinus & $70 \mathrm{~Gy} / 35 \mathrm{fr}$ & including RPLN area & $27 \mathrm{~Gy}$ \\
\hline 68 & 48 & 0 & 1 & $2 \mathrm{c}$ & 0 & $4 \mathrm{a}$ & Piriform sinus & 70Gy/35fr & including RPLN area & 28Gy \\
\hline 69 & 84 & 2 & 2 & $2 c$ & 0 & $4 a$ & Posterior wall & $70 \mathrm{~Gy} / 35 \mathrm{fr}$ & excluding RPLN area & 12Gy \\
\hline 70 & 64 & 0 & 2 & $2 \mathrm{~b}$ & 0 & $4 \mathrm{a}$ & Piriform sinus & $70 \mathrm{~Gy} / 35 \mathrm{fr}$ & including RPLN area & 34Gy \\
\hline 71 & 68 & 0 & 1 & 0 & 0 & 1 & Posterior wall & 70Gy/35fr & excluding RPLN area & 9Gy \\
\hline 72 & 65 & 0 & 2 & 1 & 0 & 2 & Piriform sinus & $70 \mathrm{~Gy} / 35 \mathrm{fr}$ & excluding RPLN area & 5 Gy \\
\hline 73 & 74 & 0 & 2 & $2 c$ & 0 & $4 a$ & Piriform sinus & $70 \mathrm{~Gy} / 35 \mathrm{fr}$ & including RPLN area & $37 \mathrm{~Gy}$ \\
\hline 74 & 71 & 0 & 2 & $2 b$ & 0 & $4 a$ & Posterior wall & 70Gy/35fr & including RPLN area & $37 \mathrm{~Gy}$ \\
\hline 75 & 72 & 0 & 2 & $2 c$ & 0 & $4 \mathrm{a}$ & Postcricoid area & 70Gy/35fr & including RPLN area & 31Gy \\
\hline
\end{tabular}




\section{Global Journal of Otolaryngology}

\begin{tabular}{|l|l|l|l|l|l|l|l|l|l|l|}
\hline 76 & 78 & 0 & 2 & 1 & 0 & 3 & Piriform sinus & $70 \mathrm{~Gy} / 35 \mathrm{fr}$ & including RPLN area & $41 \mathrm{~Gy}$ \\
\hline 77 & 61 & 0 & 2 & 0 & 0 & 2 & Posterior wall & $70 \mathrm{~Gy} / 35 \mathrm{fr}$ & including RPLN area & $42 \mathrm{~Gy}$ \\
\hline 78 & 75 & 0 & 2 & 1 & 0 & 2 & Piriform sinus & $70 \mathrm{~Gy} / 35 \mathrm{fr}$ & including RPLN area & $38 \mathrm{~Gy}$ \\
\hline 79 & 67 & 1 & 2 & 0 & 0 & 2 & Posterior wall & $66 \mathrm{~Gy} / 33 \mathrm{fr}$ & excluding RPLN area & $14 \mathrm{~Gy}$ \\
\hline
\end{tabular}

RPLN, retropharyngeal lymph node

Overall survival probabilities at 5 years for patients irradiated to the whole neck, including the RPLN area, and those excluding the RPLN area were $71 \%$ and $63 \%$, respectively (Figure 2). The difference in overall survival probabilities between patients irradiated to the whole neck, including the RPLN area, and those excluding the RPLN area was not statistically significant $(\mathrm{p}=0.314)$. Progression-free survival probabilities at 5 years for patients irradiated to the whole neck, including the RPLN area, and those excluding the RPLN area were $45 \%$ and $52 \%$, respectively, and there was no statistically significant difference $(\mathrm{p}=0.674)$ (Table 4) and (Figure 3).

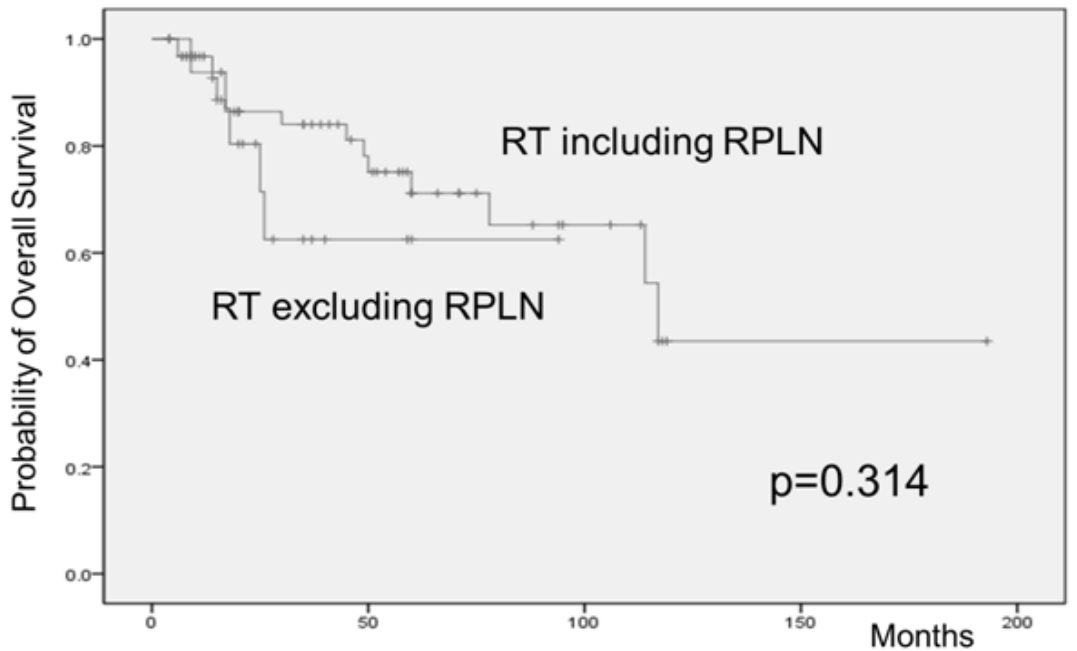

Figure 2: Overall survival probabilities at 5 years for patients irradiated to the whole neck, including the RPLN area, and those excluding the RPLN area. Kaplan-Meier analysis demonstrating no significant difference in overall survival between patients irradiated to the whole neck, including the RPLN area, and those excluding the RPLN area $(p=0.314)$. The 5 -year progression-free survival probabilities in patients irradiated to the whole neck, including the RPLN area, and those excluding the RPLN area were $71 \%$ and $63 \%$, respectively.

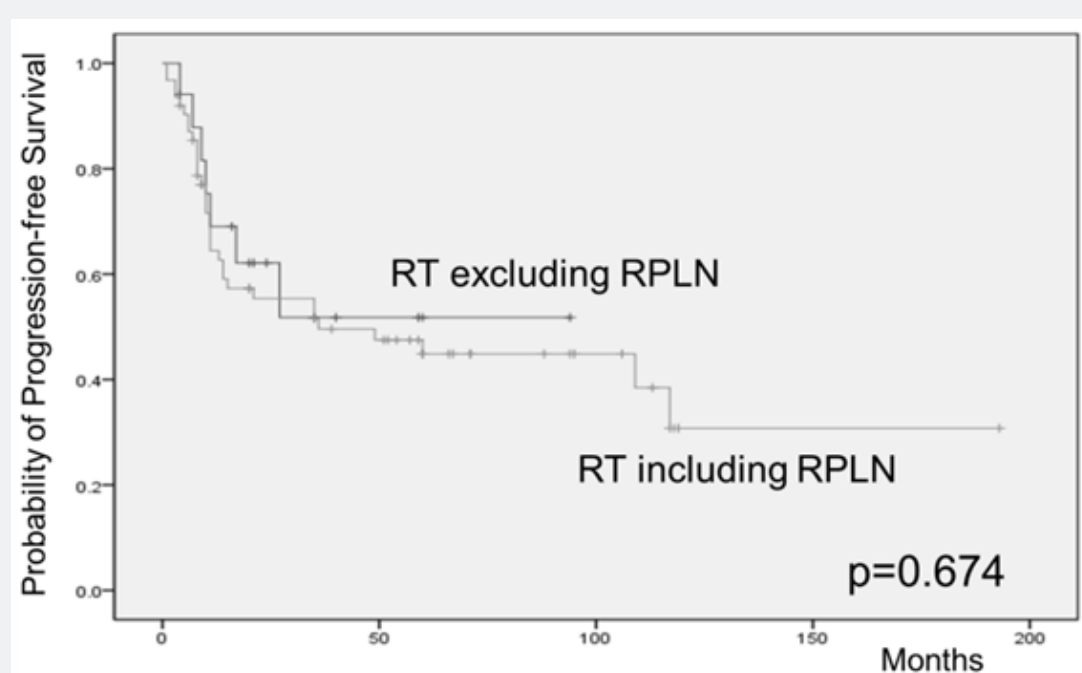

Figure 3: Progression-free survival probabilities at 5 years for patients irradiated to the whole neck, including the RPLN area, and those excluding the RPLN area. Kaplan-Meier analysis demonstrating no significant difference in progression-free survival between patients irradiated to the whole neck, including the RPLN area, and those excluding the RPLN area $(p=0.674)$. The 5 -year progression-free survival probabilities in patients irradiated to the whole neck, including the RPLN area, and those excluding the RPLN area were $45 \%$ and $52 \%$, respectively. 


\section{Global Journal of Otolaryngology}

Table 4: Chemotherapy and overall, progression-free survival for all cases.

\begin{tabular}{|c|c|c|c|c|c|c|c|}
\hline No & $\begin{array}{c}\text { Inductuin } \\
\text { chemotherapy }\end{array}$ & $\begin{array}{l}\text { Concomitant } \\
\text { chemotherapy }\end{array}$ & Desease state & $\begin{array}{l}\text { Recurremce } \\
\text { site }\end{array}$ & $\begin{array}{l}\text { Progression } \\
\text { free duration } \\
\text { (M) }\end{array}$ & Patient state & $\begin{array}{c}\text { Overall } \\
\text { survival } \\
\text { duration }(\mathrm{M})\end{array}$ \\
\hline 1 & $\begin{array}{c}\text { DOC, CDDP, } \\
5-\mathrm{FU}\end{array}$ & - & control & - & 193 & alive & 193 \\
\hline 2 & - & DOC, CDDP, 5-FU & control & - & 117 & alive & 117 \\
\hline 3 & - & DOC & control & - & 88 & alive & 88 \\
\hline 4 & - & DOC & control & - & 94 & alive & 94 \\
\hline 5 & - & - & recurrence & primary & 6 & alive & 7 \\
\hline 6 & - & DOC & control & - & 113 & alive & 113 \\
\hline 7 & - & DOC & recurrence & $\begin{array}{l}\text { primary,lymph } \\
\text { node }\end{array}$ & 109 & death & 117 \\
\hline 8 & - & DOC & recurrence & primary & 4 & death & 9 \\
\hline 9 & - & CDDP, 5-FU & recurrence & distant (liver) & 3 & death & 6 \\
\hline 10 & - & DOC, CDDP, 5-FU & control & - & 49 & death & 49 \\
\hline 11 & - & CDDP, 5-FU & control & - & 114 & death & 114 \\
\hline 12 & - & DOC, CDDP, 5-FU & control & - & 51 & alive & 51 \\
\hline 13 & - & - & control & - & 60 & death & 60 \\
\hline 14 & - & DOC & recurrence & lymph node & 35 & death & 78 \\
\hline 15 & - & - & recurrence & primary & 13 & alive & 19 \\
\hline 16 & - & DOC, CDDP, 5-FU & recurrence & primary & 5 & alive & 10 \\
\hline 17 & $\begin{array}{c}\text { DOC, CDDP, } \\
5-\mathrm{FU}\end{array}$ & CDDP & control & - & 119 & alive & 119 \\
\hline 18 & - & DOC, CDDP, 5-FU & control & - & 35 & alive & 35 \\
\hline 19 & - & DOC, CDDP, 5-FU & control & - & 95 & alive & 95 \\
\hline 20 & - & DOC, CDDP, 5-FU & control & - & 118 & alive & 118 \\
\hline 21 & - & DOC, CDDP, 5-FU & control & - & 106 & alive & 106 \\
\hline 22 & - & CDDP & recurrence & lymph node & 7 & alive & 9 \\
\hline 23 & $\begin{array}{l}\text { DOC, CDDP, } \\
5-\mathrm{FU}\end{array}$ & DOC & recurrence & $\begin{array}{c}\text { primary,lymph } \\
\text { node }\end{array}$ & 9 & death & 14 \\
\hline 24 & $\begin{array}{c}\text { DOC, CDDP, } \\
5-\mathrm{FU}\end{array}$ & DOC, CDDP, 5-FU & recurrence & lymph node & 3 & death & 15 \\
\hline 25 & - & DOC, CDDP, 5-FU & recurrence & lymph node & 1 & death & 45 \\
\hline 26 & - & - & control & - & 57 & alive & 57 \\
\hline 27 & - & DOC, CDDP, 5-FU & control & - & 60 & alive & 60 \\
\hline 28 & - & DOC, CDDP, 5-FU & control & - & 59 & alive & 59 \\
\hline 29 & - & DOC & recurrence & lymph node & 35 & death & 50 \\
\hline 30 & - & CDDP & control & - & 39 & alive & 39 \\
\hline 31 & - & DOC, CDDP, 5-FU & recurrence & primary & 14 & alive & 58 \\
\hline 32 & - & DOC, CDDP, 5-FU & control & - & 71 & alive & 71 \\
\hline 33 & - & - & recurrence & $\begin{array}{l}\text { primary,lymph } \\
\text { node }\end{array}$ & 1 & alive & 46 \\
\hline 34 & - & - & control & - & 94 & alive & 94 \\
\hline 35 & $\begin{array}{c}\text { DOC, CDDP, } \\
5-\mathrm{FU}\end{array}$ & DOC, CDDP, 5-FU & recurrence & $\begin{array}{l}\text { lymph node, } \\
\text { distant(skin) }\end{array}$ & 11 & alive & 11 \\
\hline 36 & - & - & control & - & 24 & alive & 24 \\
\hline 37 & $\begin{array}{c}\text { DOC, } \mathrm{CDDP}, \\
5-\mathrm{FU}\end{array}$ & - & recurrence & $\begin{array}{l}\text { primary,lymph } \\
\text { node }\end{array}$ & 8 & alive & 8 \\
\hline 38 & - & DOC, CDDP, 5-FU & recurrence & $\begin{array}{l}\text { primary,lymph } \\
\text { node }\end{array}$ & 11 & alive & 75 \\
\hline
\end{tabular}


Global Journal of Otolaryngology

\begin{tabular}{|c|c|c|c|c|c|c|c|}
\hline 39 & - & - & recurrence & lymph node & 9 & death & 18 \\
\hline 40 & - & DOC, CDDP, 5-FU & control & - & 54 & alive & 54 \\
\hline 41 & $\begin{array}{l}\text { DOC, CDDP, } \\
\text { 5-FU }\end{array}$ & DOC, CDDP, 5-FU & control & - & 6 & death & 6 \\
\hline 42 & - & - & control & - & 71 & alive & 71 \\
\hline 43 & - & DOC, CDDP, 5-FU & control & - & 43 & alive & 43 \\
\hline 44 & - & DOC, CDDP, 5-FU & control & - & 4 & alive & 4 \\
\hline \multirow[t]{2}{*}{45} & - & DOC, CDDP, 5-FU & recurrence & $\begin{array}{c}\text { primary,lymph } \\
\text { node, }\end{array}$ & 11 & death & 17 \\
\hline & & & & distant(bone) & & & \\
\hline 46 & - & - & recurrence & primary & 10 & alive & 16 \\
\hline 47 & - & - & control & - & 9 & alive & 9 \\
\hline 48 & $\begin{array}{l}\text { DOC, CDDP, } \\
\text { 5-FU }\end{array}$ & DOC, CDDP, 5-FU & recurrence & $\begin{array}{c}\text { primary,lymph } \\
\text { node }\end{array}$ & 8 & alive & 9 \\
\hline 49 & - & DOC, CDDP, 5-FU & control & - & 66 & alive & 66 \\
\hline 50 & - & - & control & - & 59 & alive & 59 \\
\hline 51 & - & DOC, CDDP, 5-FU & control & - & 60 & alive & 60 \\
\hline 52 & - & - & control & - & 60 & alive & 60 \\
\hline 53 & - & - & recurrence & $\begin{array}{l}\text { primary,lymph } \\
\text { node }\end{array}$ & 8 & death & 14 \\
\hline 54 & - & DOC, CDDP, 5-FU & control & - & 52 & alive & 52 \\
\hline 55 & - & - & recurrence & RPLN & 7 & death & 26 \\
\hline 56 & - & DOC, CDDP, 5-FU & recurrence & $\begin{array}{l}\text { lymph node, } \\
\text { distant(liver) }\end{array}$ & 36 & alive & 37 \\
\hline 57 & - & cetuximab & recurrence & lymph node & 10 & alive & 28 \\
\hline 58 & - & - & control & - & 40 & alive & 40 \\
\hline 59 & $\begin{array}{l}\text { DOC, CDDP, } \\
\text { 5-FU }\end{array}$ & DOC, CDDP, 5-FU & recurrence & lymph node & 21 & alive & 41 \\
\hline 60 & - & DOC, CDDP, 5-FU & recurrence & primary & 10 & death & 30 \\
\hline 61 & $\begin{array}{l}\text { DOC, CDDP, } \\
\text { 5-FU }\end{array}$ & cetuximab & recurrence & $\begin{array}{l}\text { lymph node, } \\
\text { distant(bone) }\end{array}$ & 11 & death & 15 \\
\hline 62 & - & cetuximab & control & - & 20 & alive & 20 \\
\hline 63 & $\begin{array}{l}\text { DOC, CDDP, } \\
5-\mathrm{FU}\end{array}$ & - & recurrence & $\begin{array}{c}\text { primary,lymph } \\
\text { node }\end{array}$ & 8 & alive & 35 \\
\hline 64 & - & - & recurrence & lymph node & 11 & death & 25 \\
\hline 65 & $\begin{array}{l}\text { DOC, CDDP, } \\
\text { 5-FU }\end{array}$ & cetuximab & recurrence & primary & 27 & alive & 37 \\
\hline 66 & $\begin{array}{l}\text { DOC, CDDP, } \\
\text { 5-FU }\end{array}$ & cetuximab & control & - & 35 & alive & 35 \\
\hline 67 & $\begin{array}{l}\text { DOC, CDDP, } \\
\text { 5-FU }\end{array}$ & CDDP & control & - & 20 & alive & 20 \\
\hline 68 & $\begin{array}{l}\text { DOC, CDDP, } \\
\text { 5-FU }\end{array}$ & cetuximab & recurrence & $\begin{array}{l}\text { primary,lymph } \\
\text { node }\end{array}$ & 14 & alive & 20 \\
\hline 69 & - & - & control & - & 17 & death & 17 \\
\hline 70 & $\begin{array}{l}\text { DOC, CDDP, } \\
5-\mathrm{FU}\end{array}$ & cetuximab & control & - & 20 & alive & 20 \\
\hline 71 & - & - & control & - & 21 & alive & 21 \\
\hline 72 & - & - & control & - & 16 & alive & 16 \\
\hline 73 & - & CDDP & recurrence & distant(protid) & 15 & alive & 15 \\
\hline 74 & $\begin{array}{l}\text { DOC, CDDP, } \\
\text { 5-FU }\end{array}$ & CDDP & recurrence & lymph node & 10 & alive & 14 \\
\hline
\end{tabular}




\begin{tabular}{|c|c|c|c|c|c|c|c|}
\hline 75 & $\begin{array}{c}\text { DOC, CDDP, } \\
\text { 5-FU }\end{array}$ & cetuximab & control & - & 9 & alive & 12 \\
\hline 76 & - & - & recurrence & $\begin{array}{c}\text { primary,lymph } \\
\text { node }\end{array}$ & 4 & alive & 10 \\
\hline 77 & - & - & control & - & 8 & alive & 8 \\
\hline 78 & - & CDDP & control & - & 7 & alive & 7 \\
\hline 79 & - & DOC, CDDP, 5-FU & control & - & 4 & alive \\
\hline
\end{tabular}

DOC; docetaxel hydrate, CDDP; cisplatin, 5-FU; 5-fluorouracil

\section{Discussion}

In the present study, RPLN recurrence was recognized in only $1(1.2 \%)$ of the 79 patients, and the differences in overall and progression-free survival probabilities between patients irradiated to the whole neck, including the RPLN area, and those excluding the RPLN area were not statistically significant. These results suggest that irradiation of the RPLN area in hypopharyngeal cancer without pretreatment RPLN metastasis might be unnecessary. The guidelines established by Gregoire proposed that radiotherapy to RPLNs should only be performed in specific cases. They suggest that prophylactic radiotherapy is not essential for patients with hypopharyngeal cancer classified as N0 or N1 $[13,19,20]$. Zheng et al. [21] reported that none of the N0 patients were found to exhibit RPLN metastasis, and T classification and local invasion was not significantly associated with RPLN metastasis.

Irradiation to the RPLN area might increase the incidence of adverse events, such as mucositis, dermatitis, and dry mouth, etc. In particular, dry mouth does not improve once it develops. Eisbruch et al. reported that most glands receiving a mean radiation dose higher than the threshold (24Gy for unstimulated and 26 Gy for stimulated saliva) produced little saliva with no recovery over time [22]. In the present study, the median mean dose of irradiation to the parotid glands in patients irradiated to the whole neck, including the RPLN area, and those excluding the RPLN area was 37Gy (range: $24-43 \mathrm{~Gy}$ ) and 14Gy (range: 1-27Gy), respectively, and the difference was statistically significant $(\mathrm{p}<0.001)$. This result suggests that irradiation to the whole neck, excluding the RPLN area, is reduces the risk of dry mouth.

Irradiation excluding the RPLN area might increase the risk of RPLN recurrence; however, RPLN recurrence was only identified in $1(1.2 \%)$ of the 79 patients in this study. Although the patient who developed RPLN recurrence did not receive radiotherapy to the RPLN area, this patient did not undergo chemotherapy either. We consider that chemotherapy may be able to control microscopic metastasis to the RPLN, which is undetectable on laryngoscopy, CT, MRI, PET/CT, etc. In fact, most patients (72\%) in this study received chemotherapy, whereas chemotherapy was not used for the patient who demonstrated RPLN recurrence. The main limitation of this study is the possibility of bias regarding whether RPLN was included or not and whether chemotherapy was performed or not, owing to its retrospective nature. Further prospective studies are required to confirm our findings. In the future, we plan to perform prospective studies regarding RPLN metastasis in patients with hypopharyngeal cancer.

\section{Conclusion}

We demonstrated that RPLN recurrence in patients with hypopharyngeal cancer after radiotherapy is rare $(1.2 \%)$, and the differences in overall and progression-free survival probabilities between patients irradiated to the whole neck, including the RPLN, and those excluding the RPLN area were not statistically significant. This indicates the possibility that irradiation to the RPLN area may be unnecessary in patients with hypopharyngeal cancer without pretreatment RPLN metastasis.

\section{References}

1. Cooper JS, Porter K, Mallin K, Hoffman HT, Weber RS, et al. (2009) National Cancer Database report on cancer of the head and neck: 10 year update. Head Neck 31(6): 748-758.

2. Day D, Hansen AR, Siu LL (2016) Hypopharyngeal cancer: looking back, moving forward. Curr Oncol 23(4): 221-222.

3. Takes RP, Strojan P, Silver CE, Bradley PJ, Haigentz M, et al. (2012) Current trends in initial management of hypopharyngeal cancer: the declining use of open surgery. Head Neck 34(2): 270-281.

4. Cmelak AJ, Li S, Goldwasser MA, Murphy B, Cannon M, et al. (2007) Phase II trial of chemoradiation for organ preservation in resectable stage III or IV squamous cell carcinomas of the larynx or oropharynx: results of Eastern Cooperative Oncology Group Study E2399. J Clin Oncol 25(25): 3971-3977.

5. Guadagnolo BA, Haddad RI, Posner MR, Weeks L, Wirth LJ, et al (2005) Organ preservation and treatment toxicity with induction chemotherapy followed by radiation therapy or chemoradiation for advanced laryngeal cancer. Am J Clin Oncol 28(4): 371-378.

6. Hall SF, Groome PA, Irish J, O'Sullivan B (2008) The natural history of patients with squamous cell carcinoma of the hypopharynx. Laryngoscope 118(8): 1362-1371.

7. Spector JG, Sessions DG, Haughey BH, Chao KS, Simpson J, et al. (2001) Delayed regional metastases, distant metastases, and second primary malignancies in squamous cell carcinomas of the larynx and hypopharynx. Laryngoscope 111(6): 1079-1087.

8. Yoshimoto S, Kawabata K (2005) Retropharyngeal node dissection during total pharyngolaryngectomy for hypopharyngeal cancer. Auris Nasus Larynx 32(2): 163-167.

9. Amatsu M, Mohri M, Kinishi M (2001) Significance of retropharyngeal node dissection at radical surgery for carcinoma of the hypopharynx and cervical esophagus. Laryngoscope 111(6): 1099-1103. 
10. Kamiyama R, Saikawa M, Kishimoto S (2009) Significance of retropharyngeal lymph node dissection in hypopharyngeal cancer. Jpn J Clin Oncol 39(10): 632-637.

11. Devita VT, TS L, SA R (2008) Cancer: principle \& practice of oncology. $8^{\text {th }}$ (edn.). New York: Lippincott Williams \& Wilkins.

12. Halperin EC, CA P, Brady (2008) Principles and practice of radiation oncology. ( $5^{\text {th }}$ edn). Lippincott Williams \& Wilkins, New York, USA.

13. Gregoire V, Levendag P, Ang KK, Bernier J, Braaksma M, et al. (2003) CTbased delineation of lymph node levels and related CTVs in the nodenegative neck: DAHANCA, EORTC, GORTEC, NCIC,RTOG consensus guidelines. Radiother Oncol 69(3): 227-236.

14. Tang L, Li L, Mao Y, Liu L, Liang S, et al. (2008) Retropharyngeal lymph node metastasis in nasopharyngeal carcinoma detected by magnetic resonance imaging: prognostic value and staging categories. Cancer 113(2): $347-354$

15. Zheng XK, Chen LH, Wang QS, Wu HB, Wang HM, Chen YQ et al. (2007) Influence of FDG-PET on computed tomography-based radiotherapy planning for locally recurrent nasopharyngeal carcinoma. Int J Radiat Oncol Biol Phys 69(5): 1381-1388.

16. Hickeson M, Yun M, Matthies A, Zhuang H, Adam LE, et al. (2002) Use of a corrected standardized uptake value based on the lesion size on CT permits accurate characterization of lung nodules on FDG-PET. Eur J Nucl Med Mol Imaging 29(12): 1639-1647.
17. Paulino AC, Johnstone PA (2004) FDG-PET in radiotherapy treatment planning: Pandora's box? Int J Radiat Oncol Biol Phys. United States 59(1): 4-5.

18. Fukui MB, Blodgett TM, Snyderman CH, Johnson JJ, Myers EN, et al. (2005) Combined PET-CT in the head and neck: part 2. Diagnostic uses and pitfalls of oncologic imaging. Radiographics 25(4): 913-930.

19. Gregoire V, Coche E, Cosnard G, Hamoir M, Reychler H (2000) Selection and delineation of lymph node target volumes in head and neck conformal radiotherapy. Proposal for standardizing terminology and procedure based on the surgical experience. Radiother Oncol 56(2): 135-150.

20. Gregoire V, Eisbruch A, Hamoir M, Levendag P (2006) Proposal for the delineation of the nodal CTV in the node-positive and the postoperative neck. Radiother Oncol 79(1): 15-20.

21. Wu Z, Deng XY, Zeng RF, Su Y, Gu MF, et al. (2013) Analysis of risk factors for retropharyngeal lymph node metastasis in carcinoma of the hypopharynx. Head Neck 35(9): 1274-1277.

22. Eisbruch A, Ten Haken RK, Kim HM, Marsh LH, Ship JA (1999) Dose, volume, and function relationships in parotid salivary glands following conformal and intensity-modulated irradiation of head and neck cancer. Int J Radiat Oncol Biol Phys 45(3): 577-587.

\section{Your next submission with Juniper Publishers will reach you the below assets}

- Quality Editorial service

- Swift Peer Review

- Reprints availability

- E-prints Service

- Manuscript Podcast for convenient understanding

- Global attainment for your research

- Manuscript accessibility in different formats

( Pdf, E-pub, Full Text, Audio)

- Unceasing customer service

Track the below URL for one-step submission

https://juniperpublishers.com/online-submission.php 